\title{
PRESSÃO INTRAOCULAR, PRESSÃO ARTERIAL MÉDIA E DIÂMETRO PUPILAR EM COELHOS ( (Oryctolagus cuniculus)) SUBMETIDOS AO BLOQUEIO RETROBULBAR COM DIFERENTES PROTOCOLOS ANESTÉSICOS
}

\author{
INTRAOCULAR PRESSURE, MEAN ARTERIAL BLOOD PRESSURE AND \\ PUPILLARY DIAMETER IN RABBITS ( (Oryctolagus cuniculus) ) SUBJECTED \\ TO RETROBULBAR BLOCK WITH DIFFERENT ANESTHETIC PROTOCOLS
}

\author{
Renata Maria Monção da Silva ${ }^{1}$ \\ Francisco de Assis Dórea Neto ${ }^{2}$ \\ Vivian Fernanda Barbosa ${ }^{2}$ \\ Newton Nunes3 \\ Emanoel Ferreira Martins Filho \\ Arianne Pontes Oria ${ }^{*}$
}

${ }^{1}$ Pós-graduanda da Universidade Federal da Bahia, Salvador, BA, Brasil

${ }^{2}$ Professores Doutores da Universidade Federal da Bahia, Salvador, BA, Brasil.

${ }^{3}$ Professor Doutor da Universidade Estadual Paulista, Jaboticabal, SP, Brasil

*Autora para correspondência - arianneoria@ufba.br

\section{Resumo}

O objetivo deste estudo foi buscar novos protocolos de anestesia loco regional para procedimentos oftálmicos que proporcionem segurança e manutenção das funções vitais, além de manter a pressão intraocular estável, com centralização do bulbo do olho e acinesia palpebral. Foram utilizados 20 coelhos da raça Nova Zelândia para a realização de quatro protocolos de anestesia local através do bloqueio retrobulbar com lidocaína $2 \%$ com vasoconstritor, lidocaína $2 \%$ sem vasoconstritor associada ao tramadol, ropivacaína $1 \%$ e bupivacaína $0,5 \%$, cada animal recebeu o volume anestésico de $1,0 \mathrm{~mL}$. Todos os protocolos anestésicos utilizados promoveram acinesia palpebral e centralização do bulbo do olho durante todo o período de avaliação. A realização do bloqueio retrobulbar com os protocolos anestésicos demonstrou ser factível e segura quanto à manutenção da pressão intraocular, pressão arterial invasiva e diâmetro pupilar e pode ser utilizada para realização de cirurgias intraoculares. Os anestésicos proporcionaram bom bloqueio retrobulbar, entretanto a bupivacaína foi o anestésico que ocasionou o maior diâmetro pupilar comparativamente aos demais fármacos testados.

Palavras-Chave: coelho; lidocaína; ropivacaína; tramadol.

\begin{abstract}
The aim of this study was to evaluate four protocols of loco regional anesthesia for ophthalmic procedures that could provide safety and life support, in addition to maintain intraocular pressure stable, with eye centralization and eyelid akinesia. $20 \mathrm{New}$ Zealand rabbits were used to perform local anesthesia by retrobulbar block with four protocols: $2 \%$ lidocaine with epinephrine, $2 \%$ lidocaine without epinephrine associated with tramadol, $1 \%$ ropivacaine and bupivacaine $0.5 \%$. Each animal received an anesthetic volume of $1 \mathrm{~mL}$. All anesthetic protocols used promoted eyelid akinesia and
\end{abstract}


centralization of the eye during the assessment period. The retrobulbar block with the proposed anesthetic protocols proved to be feasible and safe for the maintenance of intraocular pressure, invasive blood pressure and pupillary diameter and can be used in intraocular surgeries, respecting the time of action of each anesthetic. All protocols showed an excellent blockage action but bupivacaine promoted the highest pupil diameter compared to the other drugs tested.

Keywords: lidocaine; rabbit; ropivacaine; tramadol.

Recebido em: 13 fevereiro 2014

Aceito em: 10 junho 2015

\section{Introdução}

O bloqueio retrobulbar foi utilizado pela primeira vez em seres humanos em 1936 quando uma solução de procaína foi administrada dentro do cone muscular formado pelos músculos extraoculares ${ }^{(1)}$. A referida técnica proporciona bloqueio sensitivo, com quase nenhuma interferência sobre as funções cardiovascular e respiratória ${ }^{(2)}$. O uso dos bloqueios regionais em cirurgias oculares provoca uma amplificação da analgesia, acinesia do bulbo do olho e destaca-se pelo seu baixo custo ${ }^{(3)}$.

Em Medicina Veterinária, notadamente na área de pequenos animais o emprego de técnicas como a anestesia retrobulbar para a realização de cirurgias oftálmicas, devido à pouca colaboração dos pacientes, não são viáveis sem a associação com a anestesia geral ${ }^{(4)}$, diferentemente do que ocorre em Medicina, na qual estes procedimentos são realizados apenas com o uso de anestesia local ${ }^{(5)}$.

As diferentes técnicas de anestesia local representam alternativa efetiva e prática em muitas situações envolvendo cães, gatos e animais de outras espécies e contribuem para a anestesia cirúrgica, principalmente em animais que apresentam algum fator de risco, uma vez que a sua utilização, antes ou durante os procedimentos cirúrgicos, diminuem as doses de outros fármacos e promovem uma anestesia multimodal ${ }^{(6)}$. O uso de anestésicos locais para a realização de bloqueio retrobulbar pode se tornar uma alternativa de grande utilidade para a realização de cirurgias oftálmicas em pequenos animais, porquanto ao complementar a anestesia inalatória, permitirá que os planos anestésicos sejam mais superficiais reduzindo os riscos inerentes à conduta anestésica. Tal método deverá resultar em centralização e imobilização do bulbo do olho e, desta forma, dispensará o uso de outras medicações para esta finalidade.

O objetivo deste estudo foi avaliar quatro protocolos de anestesia local com ropivacaína 1\%, bupivacaína $0,5 \%$, lidocaína $2 \%$ sem vasoconstrictor associada ao tramadol e lidocaína $2 \%$ com vasoconstrictor em coelhos submetidos a bloqueio retrobulbar e estudar a pressão intraocular, acinesia palpebral e pressão arterial invasiva, assim como verificar o diâmetro pupilar.

\section{Material e Métodos}

Os protocolos da pesquisa foram aprovados pela Comissão de Ética no uso de Animais da Universidade Estadual Paulista "Júlio de Mesquita Filho", campus Jaboticabal, sob protocolo no 024419/11.

Foram utilizados 20 animais, adultos, machos, da raça Nova Zelândia, com pesos entre 1,9 e 2,9 kg. Todos os animais selecionados estavam isentos de alterações clínicas. Visando-se excluir animais com oftalmopatias, todos foram submetidos à avaliação oftálmica de rotina, incluindo teste lacrimal de Schirmer, tonometria de aplanação e teste de fluoresceína. Todos os animais foram mantidos em gaiolas em sala climatizada, com ração comercial e água à vontade. Os animais foram distribuídos em cinco grupos compostos por quatro animais submetidos a bloqueio retrobulbar com os anestésicos: grupo 1 com ropivacaína $1 \%$ (Ropi $^{\circledR}$, Cristália, Brasil) $2,0 \mathrm{mg} / \mathrm{kg}$; grupo $2 \mathrm{com}$ bupivacaína $0,5 \%$ (Neocaína ${ }^{\circledR}$, Cristália, Brasil) 2,0 mg/kg; grupo 3 com lidocaína $2 \%$ com vasoconstrictor (Xylestesin ${ }^{\circledR} 2 \%$ com epinefrina, Cristália, Brasil) 7,0 mg/kg; grupo 4 com lidocaína 2\% sem vasoconstrictor (Xylestesin ${ }^{\circledR}$ $2 \%$, Cristália, Brasil) $5,0 \mathrm{mg} / \mathrm{kg}$ associada ao tramadol $4,0 \mathrm{mg} / \mathrm{kg}$; e grupo 5, considerado controle, que recebeu $1,0 \mathrm{~mL}$ de solução de $\mathrm{NaCl}$ a $0,9 \%$. Após o cálculo das doses, os fármacos, sempre que 
necessário, foram diluídos em solução de $\mathrm{NaCl} 0,9 \%$, de modo que o volume final administrado fosse de 1,0 mL para todos os grupos.

A indução da anestesia foi realizada com a utilização de cetamina (Vetanarcol $®$, Konig, Brasil) $45 \mathrm{mg} / \mathrm{kg}$ e acepromazina (Acepram ${ }^{\circledR} 1 \%$, Vetnil, São Paulo, Brasil), 0,2 mg/kg, ambas por via intramuscular. Procedeu-se a tricotomia da região periocular esquerda. A manutenção anestésica foi realizada com isofluorano (Isoflurane ${ }^{\circledR}$, Cristália, São Paulo, Brasil) diluído em oxigênio, em sistema avalvular sem absorvedor com o uso de máscara nasoral. Em seguida, foi realizada a preparação do campo operatório mediante antissepsia envolvendo a lavagem do saco conjuntival com solução de polivinilpirrolidona-iodo (PVPI) diluída na proporção de 1:50 em solução de $\mathrm{NaCl}$ a $0,9 \%$.

Após a estabilização dos animais em plano satisfatório de anestesia, foram posicionados em decúbito lateral direito e o bloqueio retrobulbar foi realizado no olho esquerdo. Inseriu-se $1,0 \mathrm{~cm}$ de uma agulha $25 \mathrm{G}$ perpendicular à pele no canto medial do olho e em direção ao espaço retrobulbar. Após aspiração negativa de sangue ou humor vítreo, foi injetado $1,0 \mathrm{~mL}$ do anestésico local.

Imediatamente após o bloqueio anestésico, avaliou-se a pressão intraocular com tonômetro de aplanação (Tonopen XL), assim como acinesia palpebral, centralização e imobilização do bulbo do olho em relação ao olho contralateral, o qual permaneceu semirotacionado. O diâmetro pupilar foi mensurado através de paquímetro de Castroviejo (Petrovich Instrumentos Cirúrgicos). A aferição da pressão arterial foi realizada com auxílio de manômetro, que estava conectado ao circuito preenchido com heparina diluída em solução fisiológica e adaptado ao cateter posicionado na artéria auricular.

A pressão intraocular foi avaliada previamente à indução anestésica, decorridos 10 minutos da indução, imediatamente após o bloqueio retrobulbar (M0) e aos cinco (M1), 10 (M2) e 15 (M3) minutos subsequentes à realização do bloqueio. Os parâmetros diâmetro pupilar e pressão arterial média começaram a ser mensurados após a indução anestésica e também foram avaliados após a realização do bloqueio nos mesmos momentos que a pressão intraocular (PIO).

Os dados referentes à pressão intraocular, pressão arterial e diâmetro pupilar, obtidos na fase experimental, foram submetidos à análise estatística pelo programa de computador IBM SPSS Statistics for Windows, Version 21.0. O teste de normalidade utilizado foi o de Kolmogorov-Smirnov para verificar a distribuição normal dos dados, que foram apresentados com médias \pm desvios-padrão. Para detectar diferença das médias dentro do grupo ao longo do tempo e entre os grupos foi utilizada a análise de variância (ANOVA). As diferenças foram consideradas significativas quando $\mathrm{p} \leq 0.05$.

\section{Resultados e Discussão}

Os resultados referentes à pressão intraocular, pressão arterial invasiva e diâmetro pupilar estão dispostos, respectivamente, nas Tabelas 1, 2 e 3.

Tabela1: Médias e desvios-padrão da pressão intraocular (PIO) em mmHg de coelhos submetidos ao bloqueio retrobulbar com diferentes tipos de anestésicos locais

\begin{tabular}{ccccccc}
\hline Momentos & Controle & $\begin{array}{c}\text { Lidocaína 2\% } \\
\text { sem } \\
\text { vasoconstrictor } \\
\text { com tramadol }\end{array}$ & $\begin{array}{c}\text { Ropivacaína } \\
\mathbf{1 \%}\end{array}$ & $\begin{array}{c}\text { Lidocaína 2\% } \\
\text { com } \\
\text { vasoconstrictor }\end{array}$ & $\begin{array}{c}\text { Bupivacaína } \\
\mathbf{0 , 5 \%}\end{array}$ & $\begin{array}{c}\text { p- } \\
\text { value }\end{array}$ \\
\hline Antes da indução & $10,00( \pm 0,41)$ & $12,50( \pm 3,42)$ & $16,12( \pm 6,50)$ & $11,38( \pm 1,44)$ & $16,63( \pm 5,12)$ & 0,134 \\
Após a indução & $13,00( \pm 3,19)$ & $16,00( \pm 8,36)$ & $11,00( \pm 3,79)$ & $12,00( \pm 2,04)$ & $13,38( \pm 1,93)$ & 0,610 \\
M0 & $12,38( \pm 1,84)$ & $11,00( \pm 2,16)$ & $15,00( \pm 1,50)$ & $11,13( \pm 1,75)$ & $12,00( \pm 3,08)$ & 0,876 \\
M1 & $11,25( \pm 0,64)$ & $12,25( \pm 1,44)$ & $9,88( \pm 1,93)$ & $10,00( \pm 2,27)$ & $12,13( \pm 1,65)$ & 0,180 \\
M2 & $10,62( \pm 1,49)$ & $11,38( \pm 0,95)$ & $9,13( \pm 0,48)$ & $11,25( \pm 1,76)$ & $10,75( \pm 2,87)$ & 0,394 \\
M3 & $10,75( \pm 0,87)$ & $12,25( \pm 2,25)$ & $8,13( \pm 1,44)$ & $14,75( \pm 8,07)$ & $11,63( \pm 2,39)$ & 0,261 \\
\hline
\end{tabular}


Foram encontradas diferenças significativas entre os grupos ropivacaína e lidocaína 2\% com vasoconstrictor, em relação ao grupo lidocaína $2 \%$ sem vasoconstrictor associada ao tramadol, para 0 parâmetro pressão arterial logo após a indução anestésica e nos momentos 1 e 2 da avaliação, ou seja, 5 e 10 minutos após o bloqueio (Tabela 2).

Tabela 2: Médias e desvios-padrão da pressão arterial média (PAM) em mmHg de coelhos submetidos ao bloqueio retrobulbar com diferentes tipos de anestésicos locais

\begin{tabular}{ccccccc}
\hline Momentos & Controle & $\begin{array}{c}\text { Lidocaína 2\% } \\
\text { sem } \\
\text { vasoconstrictor } \\
\text { com tramadol }\end{array}$ & $\begin{array}{c}\text { Ropivacaína } \\
\mathbf{1 \%}\end{array}$ & $\begin{array}{c}\text { Lidocaína 2\% } \\
\text { com } \\
\text { vasoconstrictor }\end{array}$ & $\begin{array}{c}\text { Bupivacaína } \\
\mathbf{0 , 5 \%}\end{array}$ & $\begin{array}{c}\text { p- } \\
\text { value }\end{array}$ \\
\hline $\begin{array}{c}\text { Após a } \\
\text { indução } \\
\text { M0 }\end{array}$ & $63,50( \pm 17,99)^{\mathrm{ab}}$ & $84,25( \pm 7,14)^{\mathrm{b}}$ & $54,25( \pm 5,91)^{\mathrm{a}}$ & $54,50( \pm 6,40)^{\mathrm{a}}$ & $70,50( \pm 8,23)^{\mathrm{ab}}$ & $0,004^{*}$ \\
M1 & $69,75( \pm 22,16)$ & $65,50( \pm 18,06)$ & $44,50( \pm 9,00)$ & $59,50( \pm 15,52)$ & $52,75( \pm 19,52)$ & 0,303 \\
M2 & $56,50( \pm 10,66)^{\mathrm{a}}$ & $74,75( \pm 6,40)^{\mathrm{b}}$ & $45,50( \pm 6,61)^{\mathrm{a}}$ & $43,75( \pm 8,88)^{\mathrm{a}}$ & $60,75( \pm 8,30)^{\mathrm{ab}}$ & $0,001^{*}$ \\
M3 & $56,00( \pm 12,57)^{\mathrm{ab}}$ & $73,50( \pm 3,42)^{\mathrm{b}}$ & $40,50( \pm 6,81)^{\mathrm{a}}$ & $43,25( \pm 5,50)^{\mathrm{a}}$ & $54,75( \pm 11,35)^{\mathrm{ab}}$ & $0,001^{*}$ \\
\hline
\end{tabular}

a, b Médias seguidas de letras iguais nas linhas não diferem estatisticamente $(\mathrm{p}<0,05)$.

Foram encontradas diferenças significativas entre os grupos controle e lidocaína sem vasoconstrictor associada ao tramadol quando comparados ao grupo bupivacaína, para o parâmetro diâmetro pupilar apenas imediatamente após o bloqueio (Tabela 3).

Tabela 3: Médias e desvios-padrão do diâmetro pupilar (DP) em mm em coelhos submetidos ao bloqueio retrobulbar com diferentes tipos de anestésicos locais

\begin{tabular}{|c|c|c|c|c|c|c|}
\hline Momentos & Controle & $\begin{array}{c}\text { Lidocaína } 2 \% \\
\text { sem } \\
\text { vasoconstrictor } \\
\text { com Tramadol }\end{array}$ & $\begin{array}{c}\text { Ropivacaína } \\
1 \%\end{array}$ & $\begin{array}{c}\text { Lidocaína 2\% } \\
\text { com } \\
\text { vasoconstrictor }\end{array}$ & $\begin{array}{c}\text { Bupivacaína } \\
0,5 \%\end{array}$ & p-value \\
\hline $\begin{array}{l}\text { Após a } \\
\text { inducão }\end{array}$ & $4,75( \pm 1,32)$ & $5,25( \pm 0,50)$ & $4,88( \pm 0,25)$ & $4,75( \pm 0,87)$ & $6,13( \pm 1,03)$ & 0,192 \\
\hline M0 & $4,38( \pm 1,11)^{\mathrm{a}}$ & $4,88( \pm 0,95)^{\mathrm{a}}$ & $6,25( \pm 1,44)^{\mathrm{ab}}$ & $6,00( \pm 1,47)^{\mathrm{ab}}$ & $8,00( \pm 0,91)^{b}$ & $0,006^{*}$ \\
\hline M1 & $5,38( \pm 1,55)$ & $5,38( \pm 0,95)$ & $5,75( \pm 0,87)$ & $5,88( \pm 2,06)$ & $7,00( \pm 1,87)$ & 0,569 \\
\hline M2 & $5,50( \pm 1,47)$ & $5,88( \pm 1,03)$ & $6,50( \pm 0,91)$ & $6,13( \pm 1,97)$ & $6,75( \pm 1,26)$ & 0,691 \\
\hline M3 & $5,50( \pm 1,29)$ & $6,63( \pm 0,95)$ & $5,25( \pm 0,87)$ & $6,25( \pm 2,06)$ & $6,50( \pm 1,22)$ & 0,524 \\
\hline
\end{tabular}

a, b Médias seguidas de letras iguais nas linhas não diferem estatisticamente $(\mathrm{p}<0,05)$.

A busca por técnicas anestésicas factíveis e seguras para procedimentos oftálmicos é motivada pela necessidade de manutenção da pressão intraocular, relaxamento da musculatura extraocular, centralização do bulbo do olho e dilatação pupilar que são imprescindíveis para realização de cirurgias intraoculares. Na Medicina Veterinária de pequenos animais, os bloqueios regionais são pouco explorados, quando comparados com o uso destas técnicas em humanos ${ }^{(7,8)}$.

Há na literatura relatos de várias complicações decorrentes do bloqueio retrobulbar como, perfuração do bulbo do olho, quemose, lesões ao nervo óptico, paralisia dos músculos extraoculares, hemorragias conjuntivais, oclusão arteriovenosa retiniana e cegueira ${ }^{(9-13)}$. Durante o período da avaliação dos parâmetros pressão intraocular e diâmetro pupilar não foi observado surgimento de quemose, tão pouco de hemorragia conjuntival. Este achado corrobora os de Segato ${ }^{(14)}$, que também não relatou tais alterações ao estudar os efeitos de vários anestésicos, inclusive ropivacaína e bupivacaína no bloqueio retrobulbar em cães. Cabe ressaltar que o período de tempo de avaliação deste experimento foi curto quando comparado a outras pesquisas, ${ }^{(8,14)} \mathrm{o}$ que pode ter sido insuficiente para avaliação 
de alterações na espécie em questão. Nesta pesquisa, todos os bulbos oculares foram submetidos à enucleação após a avaliação da técnica anestésica e não foi observada perfuração em nenhum dos bulbos, hemorragia retrobulbar, assim como não foi constatada paralisia dos músculos extraoculares em nenhum dos animais durante a avaliação do bloqueio.

O bloqueio retrobulbar promoveu centralização do bulbo do olho, que proporcionou condições para realização das avaliações dos quatro protocolos anestésicos. O bloqueio motor, que foi avaliado através da centralização do bulbo ocorreu cerca de um minuto após a aplicação dos anestésicos no espaço retrobulbar em todos os grupos e teve duração média de 15 minutos.

A acinesia e centralização do bulbo do olho são requeridas para realização de cirurgias intraoculares, porém, quando os animais de pequeno porte estão em plano anestésico cirúrgico, é comum a rotação do bulbo na órbita o que impede a visualização da córnea de forma total ou parcial. Por este motivo, em geral, são utilizados bloqueadores neuromusculares para centralização do bulbo ${ }^{(15)}$. Contudo, neste estudo, não foi necessário o uso de nenhuma medicação adicional, uma vez que todos os anestésicos estudados mantiveram o bulbo do olho centralizado durante o período de avaliação.

Em procedimentos cirúrgicos oftálmicos, também é imprescindível que a PIO seja mantida dentro dos valores de normalidade ou inferiores. Vários fatores podem influenciar o aumento da PIO durante a cirurgia, como a pressão das pálpebras sobre o bulbo, a contração dos músculos extraoculares, as suturas para fixar o olho, a compressão dos afastadores de pálpebras e manobras cirúrgicas agressivas também podem levar ao aumento da pressão intraocular. ${ }^{(15)}$

Os protocolos de anestesia local utilizados nesta pesquisa não influenciaram no aumento da PIO. Sabe-se que injeção de grandes volumes de anestésicos por via retrobulbar ou peribulbar cursa com alterações significativas no fluxo sanguíneo ocular ${ }^{(16)}$, isto provoca um aumento transitório da PIO devido à elevação da pressão intraorbitária. No entanto, a PIO tende a se normalizar devido à dispersão dos fármacos e ao relaxamento dos músculos extraoculares (pós-bloqueio), fato que leva à diminuição da pressão externa sobre o bulbo do olho ${ }^{(17)}$.

Os valores médios encontrados para o parâmetro pressão intraocular foram semelhantes aos relatados na literatura para a espécie, que variam entre $10,21 \pm 1,32$ a $17,39 \pm 3,21 \mathrm{mmHg}^{(18,19)}$. Os dados aqui encontrados corroboram com a literatura que, relata que a lidocaína, a bupivacaína e a ropivacaína, quando utilizados no bloqueio retrobulbar, não causam elevação da $\mathrm{PIO}^{(20,21)}$. A ropivacaína promoveu discreta diminuição da PIO, o que pode estar relacionado com a atividade vasoconstritora intrínseca do fármaco, que foi descrita quando se estudou sua ação em capilares e vasos ${ }^{(22,23)}$; no entanto, não se devem extrapolar os dados para diferentes leitos vasculares, uma vez que cada local responde de diferentes formas frente a drogas e estímulos ${ }^{(24,25)}$. Relativo ao seu efeito vasoconstritor, a ropivacaína $1 \%$ tem maior eficácia em reduzir a PIO por promover relaxamento da musculatura extraocular e diminuir o volume sanguíneo intraocular ${ }^{(26-29)}$.

Nos últimos anos, estudos têm demonstrado a ação anestésica local do tramadol ${ }^{(30)}$. Pang et al. ${ }^{(31)}$ foram capazes de promover bloqueio sensorial ao toque, à picada de agulha e ao frio, ao injetar, por via intradérmica, solução de tramadol $5 \%$, e relataram que foi semelhante ao provocado pela lidocaína $1 \%$. O mecanismo da ação anestésica deste fármaco é desconhecido; entretanto, Acalovschi et al. ${ }^{(30)}$ relataram que o menos provável é que esta ação ocorra pela sua interação com receptores opióide periféricos.

O tramadol é um potente opióide e suas propriedades analgésicas já foram comprovadas ${ }^{(32)}$. Em estudo para avaliação de anestesia regional intravenosa a adição de $100 \mathrm{mg}$ de tramadol à solução de lidocaína, reduziu-se o início do bloqueio sensorial e o consumo de fentanil e se aumentou o tempo para aplicação de analgésicos no pós-operatório ${ }^{(32)}$. Outro estudo que utilizou o tramadol demonstrou suas propriedades anestésicas, porém apresentou menor eficácia quando comparado à lidocaína ${ }^{(33)}$. Acalovschi et al. ${ }^{(30)}$ afirmaram que a grande desvantagem do uso do tramadol como anestésico local é o aumento da ocorrência de efeitos colaterais e relataram que 15 pacientes apresentaram erupções cutâneas distais ao local da aplicação venosa do tramadol e associaram o efeito à liberação de histamina. Entretanto, neste estudo, não foi observada reação local retrobulbar com o uso do fármaco, o que pode estar relacionado à não ocorrência de lesão venosa durante a realização do bloqueio.

Há na literatura descrição do uso dos fármacos lidocaína, com e sem vasoconstritor, bupivacaína e ropivacaína, tanto em bloqueio retrobulbar quanto em peribulbar em cães e em seres humanos ${ }^{(2,8,}$ 26); contudo, não existem relatos da utilização da mistura de lidocaína sem vasoconstritor associada 
ao tramadol para esta modalidade anestésica. Relatos desta associação para bloqueio de nervos periféricos mostrando-se eficaz já foram descritos ${ }^{(34)}$.

A mensuração da pressão arterial de forma invasiva é considerada a mais fidedigna ${ }^{(35)}$ por representar a pressão sistêmica de maneira exata e consistente; entretanto, é pouco utilizada, devido às dificuldades da técnica ${ }^{(36)}$, que necessita de sedação ou anestesia do paciente, o que poderá interferir na variável avaliada ${ }^{(37)}$. Devido a estes fatores, é pouco consignada na rotina e tem maior emprego em condições de pesquisa ${ }^{(38)}$. Em Medicina Veterinária de pequenos animais, o aumento da PAM está relacionado ao aparecimento de alterações oculares como retinopatia, glaucoma, hifema e cegueira ${ }^{(39)}$.

Neste estudo, ao se compararem os valores encontrados para PAM com os fisiológicos que variam de 70 a $103 \mathrm{mmHg}^{(40)}$, pôde ser observado que apenas no grupo lidocaína sem vasoconstritor associada ao tramadol esse parâmetro foi mantido em dois momentos (M1 e M2) dentro da faixa de normalidade. Este fato pode estar relacionado à ação do tramadol sobre o sistema nervoso simpático. Em estudo conduzido em ratos, Nagaoka et al. ${ }^{(41)}$ administraram tramadol na dose 2,0 a $4,0 \mathrm{mg} / \mathrm{kg}$ IV e observaram aumento dos níveis séricos de noradrenalina, elevando de forma significativa a PAM, o que sugere que este fármaco promove ativação do sistema nervoso simpático. E possível propor, então, que, no grupo em que o tramadol foi utilizado, a PAM foi mantida dentro dos valores de normalidade nos momentos M1 e M2, pela ação inibitória de recaptação neuronal de noradrenalina e serotonina ${ }^{(42)}$, que promove o aumento destes neurotransmissores no sistema nervoso central ${ }^{(43)}$.

Neste estudo, os animais foram induzidos à anestesia com uma mistura de cetamina que, segundo os autores, é capaz de elevar a pressão arterial( ${ }^{(4,15)}$; contudo, este fármaco foi associado à acepromazina, que é um potente hipotensor ${ }^{(44)}$, e essa associação pode ter equilibrado a ação destes medicamentos na PAM. Cabe ressaltar ainda que, em quatro grupos, os valores médios encontrados para a PAM foram menores que os valores de normalidade, isso pode ser devido ao fato de a manutenção anestésica ter sido feita com isofluorano, que, segundo Dalal et al ${ }^{(45)}$, tem efeito depressor sobre a pressão arterial. A midríase total é condição amplamente desejada e necessária para a realização de procedimentos intraoculares e está consignada à ação dos músculos constritor e dilatador da pupila ${ }^{(15)}$. A bupivacaína apresentou maior poder de dilatação pupilar comparativamente aos outros anestésicos, principalmente logo após a realização do bloqueio. A utilização deste anestésico em bloqueio retrobulbar para cirurgia de catarata em humanos provocou dilatação pupilar satisfatória e a manteve durante todo o procedimento $^{(46)}$.

Como demonstrado em estudos tanto em humanos quanto em animais ${ }^{(2,8,28)}$, os anestésicos utilizados nesta pesquisa mostraram boa qualidade de bloqueio durante o período de avaliação. Desta forma, deve-se considerar a atuação da lidocaína com vasoconstritor para cirurgias rotineiras de enucleação em cães e gatos, por ser uma excelente opção, comparativamente aos outros fármacos, por promover menor sangramento no sitio operatório e possuir adequado período de ação para o procedimento proposto.

\section{Conclusão}

A realização do bloqueio retrobulbar com os protocolos anestésicos demonstrou ser factível e segura quanto à manutenção da pressão intraocular, pressão arterial invasiva e diâmetro pupilar e pode ser utilizada para a realização de cirurgias intraoculares. Os anestésicos proporcionaram bom bloqueio retrobulbar; entretanto, a bupivacaína ocasionou o maior diâmetro pupilar comparativamente aos outros fármacos testados.

\section{Agradecimento}

À Coordenação de Aperfeiçoamento de Pessoal de Nível Superior (CAPES) pela bolsa de estudos concedida e à União Metropolitana de Educação e Cultura (UNIME). 


\section{Referências}

1. Wong, DHW. Review article: Regional anaesthesia for intraocular surgery. Can. Anaesth Soc J. 1993; 40 (7): 635-657.

2. Gouveia CS, Magalhães E. Anestesia peribulbar com ropivacaína - Estudo da ação vasoconstritora. Rev Bras Anestesiol. 2010; 60 (5): 503-512.

3. Gillart T, Dualé C, Curt I. Ophthalmic regional anaesthesia. Curr Opin Anaesthesiol. 2002; 15: 503-509.

4. Nunes N, Laus JL. Técnicas anestésicas destinadas à cirurgia ocular no cão. Braz J Vet Res Anim Sci. 1995; 32 (2); 177-180.

5. Coelho RP, Weissheimer J, Romão E, Cruz AA. V. Comparação entre a dor provocada pela facoemulsificação com anestesia tópica e a pela infiltração peribulbar sem sedação. Arq Bras Oftalmol. 2005; 68 (1): 45-48.

6. Oliva VNLS, Andrade AL. Anestesia peribulbar em facectomia extracapsular em cães. Medvep. 2010; 8: 207-213.

7. Klaumann PR, Wouk AFPF, montiani-Ferreira F, Vilani, RGD'OC. Estudo comparativo entre ropivacaína e lidocaína no bloqueio peribulbar em cães. Medvep. 2008; 6: 268-277.

8. Hazra S, DE D, Roy B, Bose A, NandI S, Konar A. Use of ketamine xylazine and diazepam anesthesia with retrobulbar block for phacoemulsification in dogs. Vet Ophthalmol. 2008; 11(4): 255-259.

9. Parness G, Underhill S. Regional anaesthesia for intraocular surgery. Contin Educ Anaesth Crit Care Pain. 2005; 5 (3): 93-97.

10. Chinchurreta-Capote A, Beltrán Fernández-Ramos M. Amartínez-De-Velasco-Santos C. Contralateral amaurosis and extraocular muscle palsies after retrobulbar. Arch Soc Esp Oftalmol. 2006; 81: 45-48.

11. Kumar CM, Dodds C. Sub-Tenon's anesthesia. Ophthalmol Clin North Am. 2006; 19: 209-219.

12. Gayers, Kumar CM. Ophthalmic regional anesthesia techniques. Minerva Anestesiol. 2008; 74 (1-2): 2333.

13. Kumar C, Dowd T. Ophthalmic regional anaesthesia. Curr Opin Anaesthesiol. 2008; 21: 632-637.

14. Honsho cs, Franco LG, Cerejo SA, Segato MB, Ferreira MA, Bolzan AA, Duque CTN, Laus JL. Ocular effects of retrobulbar block with different local anesthetics in healthy dogs. Semina: Ciên Agr. 2014; 35 (5): 2577-2590.

15. Carareto R, Nunes N, Sousa MG, Ferro Pc, Guerrero PNH, Nishimori CT, Paula DP, Conceição EDV. Anestesia para Cirurgias Oftálmicas em Canídeos. Rev Port Ciên Vet. 2007; 102: 35-42.

16. Chang, BYP. Local anesthetic techniques and pulsatile ocular blood flow. Brit J Ophthalmol. 2000; 84: 1260-1263.

17. Johnson RW, Forrest FC - Anaesthesia for Ophthalmic Surgery, em: Serzedo PSM, Nociti JR, Zuccolotto EB, Cagnolati CA, Ferrera SB. Intraocular Pressure and Ropivacaine in Peribulbar Block: A Comparative Study with Bupivacaine. Acta Anaesth Scand. 2001; 45 (5): 600-602.

18. Zhang L, LI Y, Zhang C, Wang Y, Song C. Pharmacokinetics and tolerance study of intravitreal injection of dexamethasone-loaded nanoparticles in rabbits. Int J Nanomedicine. 2009; 4:175-183.

19. Pereira FQ, Bercht BS, Soares MG, da Mota MGB, Pigatto JAT. Comparison of a rebound and an applanation tonometer for measuring intraocular pressure in normal rabbits. Vet Ophthalmol. 2011; 14: 321-326.

20. Magalhães E, Govêia CS, Oliveira KB. Bupivacaína racêmica levobupivacaína e ropivacaína em anestesia locorregional para oftalmologia- um estudo comparativo. Rev Assoc Med Bras. 2004; 50 (2): 195-198.

21. Olmez G, Cakmak SS, Caca I, Unlu MK. Intraocular pressure and quality of blockade in peribulbar anesthesia using ropivacaine or lidocaine with adrenaline: a double blind randomized study. Tohoku J Exp Med. 2004; (204): 203-208.

22. Dahl JB, Simonsen L, Mogensen T, Henriksen JH, Kehlet H. The effect of $0.5 \%$ ropivacaine on epidural blood flow. Acta Anaesth Scand. 1990; 34: 308-310.

23. Cederholm I, Evers H, Lofstrom JB. Skin blood flow after intradermal injection of ropivacaine in various concentrations with and without epinephrine evaluated by laser Doppler flowmetry. Region Anesth Pain M. 
1992; 17: 322-328.

24. Kopacz DJ, Carpenter RL, Mackey DC. Effect of ropivacaine on cutaneous capillary blood flow in pigs. Anesthesiol. 1989; 71: 69-74.

25. Iida H, Watanabe Y, DOHI S, Ishiyama T. Direct effects of ropivacaine and bupivacaine on spinal pial vessels in canine: Assessment with closed spinal window technique. Anesthesiol. 1997; 87: 75-81.

26.Serzedo PSM, Nociti JR, Zuccolotto EB, Cagnolati CA, Ferreira SB. Pressão intraocular durante bloqueio peribulbar com ropivacaína a 1\%. Rev Bras Anestesiol. 2000; 50 9(3): 251-253.

27.Nociti JR, Serzedo PSM, Zuccolotto EB, Nunes AM, Ferreira SB. Intraocular pressure and ropivacaine in peribulbar block: a comparative study with bupivacaine. Acta Anaesth Scand. 2001; 45 (5): 600-602.

28.Serzedo PSM, Nociti JR, Zuccolotto EB, Cagnolati CA, Ferrera SB. Intraocular Pressure and Ropivacaine in Peribulbar Block: A Comparative Study with Bupivacaine. Acta Anaesth Scand. 2001; 45 (5): 600-602.

29.Ozcan Aa, Ozdemir N, günes Y, Bozkurt A, Yagmur M, Alparslan ZN.Intraocular pressure quality of block and degree of pain associated with ropivacaine in peribulbar block: a comparative randomized study with bupivacaine-lidocaine mixture. Eur J Ophthalmol. 2003;13 (9-10): 794-797.

30.Acalovschi I, Cristea T, Margarit S, Gavrus R. Tramadol added to lidocaine for intravenous regional anesthesia. Vet Anaesth Analg. 2001; 92: 209-214.

31.Pang WW, Mok MS, Chang DP, Huang MH. Local anesthetic effect of tramadol metoclopramide and lidocaine following intradermal injection. Region Anesth Pain M. 1998; 23: 580-583.

32.Siddiqui AK, Mowafi HA, Al-Ghamdi A, Ismail SA, Abudzeid HA. Tramadol as adjuvant to intravenous regional anesthesia with lignocaine. Saudi Med J. 2008; 9 (8):1151-1155.

33.Mert T, Gunes Y, Guven M, Gunay I, Ozcengiz D. Blocking Action of Tramadol on Nerve Conduction. Internet J Pharmacol. 2002; 1(2).

34.Mert, T.; Gunes, Y.; Guven, M.; Gunay, I. Local analgesic efficacy of tramadol following intraplantar injection. Eur J Pharmacol. 2007 v. 558, p. 68-72,.

35.Stepien RL, Rapoport GS. Clinical comparison of three methods to measure blood pressure in nonsedated dogs. J Am Vet Med Assoc. 1999; 215: 1623-1628.

36.Porciello F, Birettoni F, Conti MB, Marinetti C, Antognoni MT, Fruganti G. Blood pressure measurements in dogs and horses using the oscillometric technique: personal observations. Vet Res Commun. 2004; 28: $367-$ 369.

37.Kittleson MD, Oliver NB. Measurement of systemic arterial blood pressure. Vet Clin N Am-Small. 1983; $13: 321-336$.

38.Chalifoux A, Dallaire A, Blais D, Lariviere N, Pelletier N. Evaluation of the arterial blood pressure of dogs by two noninvasive methods. Can J Vet Res. 1985; 49: 419-423.

39.Brown S, Atkins C, Bagley R, Carr A, Cowgill L, Davidson M, Egner B, Elliott J, Henik R, Labato M, Littman M, Polzin D, Ross L, Snyder P, Stepien R. Guidelines for the identification evaluation and management of systemic hypertension in dogs and cats. J Vet Intern Med. 2007; 21 (3): 542-558.

40.Flecknell PA. Anaesthesia of animals for biomedical research. Brit J Anaesth. 1993; 71 (6): 885-94.

41.Nagaoka E,Minami K,Shiga Y,Uezono Y,Shiraishi M, Aoyama K,Shigematsu A. Tramadol has no effect on cortical renal blood flow- despite increased serum catecholamine levels- in anesthetized rats: implications foranalgesia in renal insufficiency. Vet Anaesth Analg. 2002; 94: 619-625.

42.Raffa RB, Friderichs E, Reimann W, Shank RP, Codd EE, Vaught JL. Opioid and nonopioid components independently contribute to the mechanism of action of tramadol an "atypical" opioid analgesic. J Pharmacol Exp Ther. 1992; 260 (1) 275-285.

43.Shipton EA. Tramadol - Present and future. Anaesth Intens Care. 2000; 28: 363-374.

44.Lipman NS, Marini PR, Erdman SE. A comparison of ketamine/xylazine and ketamine/xylazine/ acepromazina anesthesia in the rabbit. Lab Anim Sci. 1990; 40: 395-398.

45.Dalal PG, Corner A, Chin C, Wood C, Ravazi R.Comparison of the cardiovascular effects of isoflurane and sevoflurane as measured by magnetic resonance imaging in children with congenital heart disease. J Clin 
Anesth. 2008; 20: 40-44.

46.Gombos, K.; Jakubovits, E.; Kolos, A.; Salacz, G.; Nemeth, J. Cataract surgery anaesthesia: is topical anaesthesia really better than retrobulbar? Acta Ophthalmol Scand. 2007; 85 (3): 309-316. 